\title{
Physically-based failure models and criteria for laminated fibre-reinforced composites with emphasis on fibre kinking. Part I: Development
}

\author{
S T Pinho*, L Iannucci and P Robinson \\ Department of Aeronautics, South Kensington Campus, Imperial College London, \\ SW7 2AZ, London, U.K.
}

\begin{abstract}
3D failure criteria for laminated fibre-reinforced composites, based on a physical model for each failure mode and considering nonlinear matrix shear behaviour, are developed. Special emphasis is given to compression failure. The physical model for matrix compression failure is based on the Mohr-Coulomb criterion and also predicts the fracture angle. For fibre kinking, an initial fibre-misalignment angle is considered to trigger failure, due to further rotation during the compressive loading. The plane where the kinking takes place is predicted by the model, as well as the kink-band angle. Applications are presented that validate the model against experimental data.
\end{abstract}

Key words: A Carbon Fibre, B Fracture, C Analytical Modelling, Failure

* Corresponding author. Tel. +4402075945107, Fax. +4402075848120
Email addresses: silvestre.pinho@imperial.ac.uk (S T Pinho),

1.iannucci@imperial.ac.uk (L Iannucci), p.robinson@imperial.ac.uk (P 


\section{Introduction}

The mechanisms that lead to failure in composite materials are not fully understood yet. This is especially true for compression failure, both for the matrix and fibre-dominated failure modes. This has become particularly evident after the World Wide Failure Exercise (WWFE) [1].

This series of two papers covers two main themes. In this first paper, physicallybased failure models are discussed and proposed for each failure mode in laminated fibre-reinforced composites with unidirectional plies, at the ply level. The failure analysis of laminated composites, considering fibre-dominated failure modes and matrix-dominated failure modes can be tracked back to Hashin $[2,3]$

Experimental results from the World Wide Failure Exercise (WWFE) [1,4] indicate that the (admittedly scarce) data on fibre tensile failure under bior multi-axial stress states does not seem to invalidate the maximum stress criterion. Thus, this paper focuses on models for compression failure, which is of great interest in crashworthiness and other areas, as well as matrix tensile failure.

In this work, accurate physically-based criteria are developed and preferred to curve-fitting-based criteria. The main limitation associated with curve-fittingbased criteria is that their applicability is restricted to the load combinations used in the curve fitting from which they originate. However, it is impractical to test every material in enough load combinations to define these criteria for every combination of the six stress tensor components.

$\overline{\text { Robinson). }}$ 
Matrix compression failure is addressed with a model based on the MohrCoulomb criterion. Puck et al. [5-8] were the first researchers to propose a matrix failure model based on the Mohr-Coulomb criterion. Further developments were later carried by Dávila et al. [9,10] for the LaRC02/03 failure criteria. In this present work, an analysis of both Puck [7,8] and LaRC02/03 $[9,10]$ matrix compression failure criteria is performed. For the LaRC02/03 criteria, a correction is proposed for the consideration of friction stresses. This leads to more conservative predictions, and makes the resulting failure envelope coincide with a simpler criterion that can be related to the work from Puck and Schürmann [7,8]. The analysis concludes with the proposal of the latter as a matrix failure criterion for a three dimensional (3D) stress state. Matrix tensile failure is addressed combining the action or fracture plane concept from Puck and Schürmann $[7,8]$ with experimental evidence from the WWFE.

For fibre failure in compression, a review [11] suggests kink-band formation results from matrix failure, due to small misalignments of the fibres in the composite. Also, the review indicates shear nonlinearity should have a considerable effect on failure and 2D analyses of fibre kinking over-simplify the treatment of the problem. In this present work, a formal treatment of fibre kinking is presented, that leads to a model for fibre kinking similar to the one proposed by Dávila et al. $[9,10]$. The main differences are that the model presented here accounts for 3D effects, considers a generic nonlinear shear behaviour, and uses the matrix failure criteria developed in this paper.

In the second paper of the series [12], the implementation of the failure models into a commercial explicit FE code [13] is described. After failure is initiated, the failure process is smeared over the finite element dimension. This allows 
for constant energy absorption, regardless of mesh refinement. The resulting FE model is shown to have captured some interesting features of the failure process.

In this paper, the index $a$ refers to the fibre direction, the index $b$ refers to the in-plane transverse direction and the index $c$ refers to the through-thethickness direction.

\section{Fibre tensile failure}

For fibre tensile failure, it is somewhat difficult to argue whether stresses other than $\sigma_{a}$ contribute to promote failure and have any influence on the strength. For instance, Soden et al. [4] obtained experimental data to define the $\left(\sigma_{a}, \tau_{a b}\right)$ failure envelope of CFRP, and the $\left(\sigma_{a}, \sigma_{b}\right)$ failure envelope of GRP. From their results, it does not seem possible to draw sound conclusions on how the different stress components interact to promote failure. Therefore the maximum stress failure criterion is used:

$$
f_{f t}=\frac{\sigma_{a}}{X_{t}}=1
$$

\section{Matrix compression failure}

\subsection{Mohr-Coulomb based criteria}

The orientation of the fracture surface of specimens failing by matrix compression suggests that the Mohr-Coulomb criterion is applicable to matrix compression failure [5-8]. Matrix compression specimens fail by shear. For a 

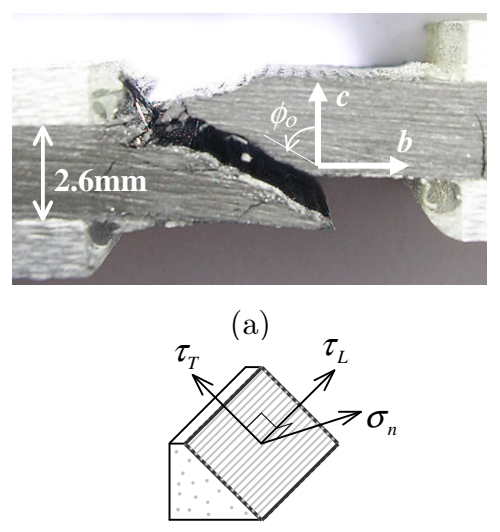

(c)
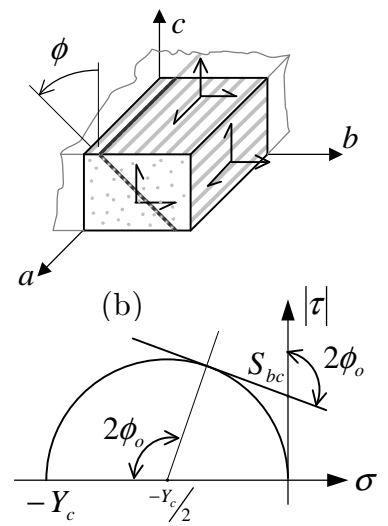

(d)

Fig. 1. (a) Pure transverse compression failure, for a CFRP specimen; (b) Fracture plane for a 3D stress state; (c) stresses in the fracture plane; (d) geometrical representation of the Mohr-Coulomb criterion

pure compression loading, this fact suggests that the angle of the fracture surface with the through-the-thickness direction should be $\phi_{o}=45^{\circ}$, i.e. fracture should occur in the plane of the maximum shear stresses. However, it is experimentally seen that the angle is generally $\phi_{o}=53 \pm 2^{\circ}$ for most technical composite materials [7,8], Fig. 1(a). This can be explained through the existence of a compressive stress acting on the potential fracture surfaces, and an associated friction stress.

The designation 'friction stress' is here used, as it was by previous authors $[7,8]$, even though there is no interface before fracture. At the micro-mechanical level, the effective macro-mechanical friction stress can be explained, at least partially, as resulting from the 'true' friction stress acting in the micro-cracks in the matrix before failure.

For a general loading situation, Fig. 1(b), the angle of the fracture plane with the through-the-thickness direction, denoted as $\phi$, might assume a different value than the one for pure compression $\left(\phi_{o}\right)$. The particular orientation of 
the fracture plane depends on the particular combination of shear $\left(\tau_{T}\right.$ and $\left.\tau_{L}\right)$ and normal $\left(\sigma_{n}\right)$ tractions for each particular value of $\phi$, Fig. 1(c).

In a $3 \mathrm{D}$ formulation, the tractions are obtained from the components of the stress tensor and the fracture plane angle $\phi$ :

$$
\begin{aligned}
& \sigma_{n}=\frac{\sigma_{b}+\sigma_{c}}{2}+\frac{\sigma_{b}-\sigma_{c}}{2} \cos (2 \phi)+\tau_{b c} \sin (2 \phi) \\
& \tau_{T}=-\frac{\sigma_{b}-\sigma_{c}}{2} \sin (2 \phi)+\tau_{b c} \cos (2 \phi) \\
& \tau_{L}=\tau_{a b} \cos (\phi)+\tau_{c a} \sin (\phi) .
\end{aligned}
$$

The Mohr-Coulomb failure criterion is expressed in terms of the tractions in the fracture plane, and can be written in several forms. Considering first the case where $\tau_{L}=0$, the Mohr-Coulomb criterion can be expressed as

$$
\left|\tau_{T}\right|+\mu_{T} \sigma_{n}=S_{T} \quad\left(\sigma_{n}<0\right)
$$

where $\mu_{T}$ is a friction coefficient and $S_{T}$ is the fracture plane fracture resistance against its fracture by transverse shear ${ }^{1}$. For simplicity, $S_{T}$ is not considered to depend on $\phi$, and will be designated as transverse shear strength. For a more detailed explanation on the difference between fracture plane fracture resistance and strength, see Ref. [7]. The geometrical representation of this criterion in a $(\sigma,|\tau|)$ space is a line with negative slope $\left(-\mu_{T}\right)$, shown in Fig. 1(d). In this figure, the Mohr-Coulomb criterion's line is tangential to the Mohr circle corresponding to the case of failure by pure compression. The slope of the Mohr-Coulomb criterion's line can be related to the angle of the $\overline{1}$ The fracture plane resistance of a potential fracture plane parallel to the fibres is the resistance of this plane against its fracture due to a single stressing acting in this plane [8]. 
fracture plane in pure compression, $\phi_{o}$, through

$$
\tan \left(2 \phi_{o}\right)=-\frac{1}{\mu_{T}} .
$$

Furthermore, writing Eq. 3 for a pure compression case establishes the relation between $S_{T}, Y_{c}$ and $\phi_{o}[14]$ :

$$
S_{T}=\frac{Y_{c}}{2 \tan \left(\phi_{o}\right)} .
$$

The angle $\phi_{o}$ can be easily determined from simple compression tests, Fig. 1(a), and allows the determination of $\mu_{T}$ and $S_{T}$ by using Eqs. 4 and 5 .

The Mohr-Coulomb criterion (Eq. 3) can be expressed in several forms, namely considering that friction affects (increases) the strength, or that it affects (decreases) the applied stress. Probably motivated by those two different forms of expressing the same criterion for $\tau_{L}=0$, Puck and Schürmann $[7,8]$ initially proposed for the general case $\left(\tau_{L} \neq 0\right)$

$$
f_{m c}=\left(\frac{\tau_{T}}{S_{T}-\mu_{T} \sigma_{n}}\right)^{2}+\left(\frac{\tau_{L}}{S_{L}-\mu_{L} \sigma_{n}}\right)^{2}=1,
$$

whereas Dávila et al. proposed first for the LaRC02 [9] failure criteria and subsequently for the LaRC03 [10] failure criteria

$$
f_{m c}=\left(\frac{\left\langle\left|\tau_{T}\right|+\mu_{T} \sigma_{n}\right\rangle}{S_{T}}\right)^{2}+\left(\frac{\left\langle\left|\tau_{L}\right|+\mu_{L} \sigma_{n}\right\rangle}{S_{L}}\right)^{2}=1
$$

where $S_{L}$ is the longitudinal shear strength (for simplicity, $S_{L}$ is considered not to depend on $\phi$ ) and the operator $\langle\cdot\rangle$ is the Mc-Cauley bracket defined by $\langle x\rangle=\max \{0, x\}, x \in \mathbb{R}$. Clearly, Puck and Schürmann (Eq. 6) consider that the compression stress $\left(\sigma_{n}\right)$ increases the effective strength, while Dávila et al. (Eq. 7) consider that the compression stress reduces the effective shear stress. Puck and Schürmann $[7,8]$ finally choose to use the following equation, 
arguing that it fits the experimental data better:

$$
f_{m c}=\frac{\left(\tau_{T}\right)^{2}}{S_{T}^{2}-2 \mu_{T} S_{T} \sigma_{n}}+\frac{\left(\tau_{L}\right)^{2}}{S_{L}^{2}-2 \mu_{L} S_{L} \sigma_{n}}=1 .
$$

For the friction coefficient $\mu_{L}$ in Eqs. 6, 7 and 8, Puck and Schürmann [7] proposed to use the following equation

$$
\frac{\mu_{L}}{S_{L}}=\frac{\mu_{T}}{S_{T}}
$$

in the absence of biaxial experimental data. This suggestion has later been considered by Dávila et al. for the LaRC02/03 criteria $[9,10]$.

\subsection{Comparison and Improvements}

The comparison of the criteria expressed in Eqs. 6, 7 and 8 is shown in Fig. 2 for a material with the properties presented in Table 1. In Fig. 2, the fracture angle for each point in each curve was determined by trying several tentative angles, as explained later in this subsection.

While the initial and final Puck criteria (Eqs. 6 and 8) yield similar results, LaRC02/03 criterion (Eq. 7) is less conservative. This is related to the fact that the effect of friction is over-estimated in Eq. 7. Indeed, affecting the shear tractions by a friction term as in Eq. 7 over-estimates the friction forces whenever both $\tau_{T}$ and $\tau_{L}$ are acting simultaneously. As Fig. 3(a) represents, supposing a very simple case with isotropic friction $\left(\mu_{T}=\mu_{L}\right)$, the friction stresses are over-estimated by a factor of $\sqrt{2}$ when using Eq. 7 .

It is interesting to notice the effect that an orthotropic friction model has on 
Table 1

Mechanical properties

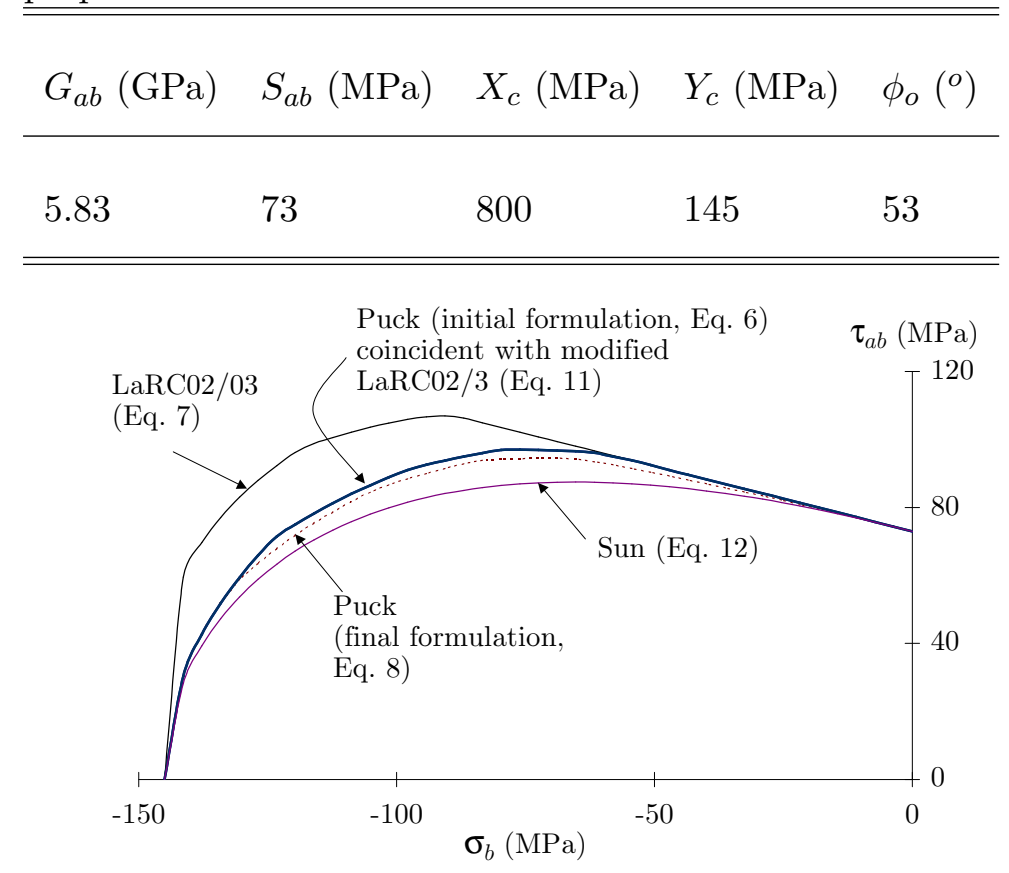

Fig. 2. Failure envelopes for transverse compression and in-plane shear

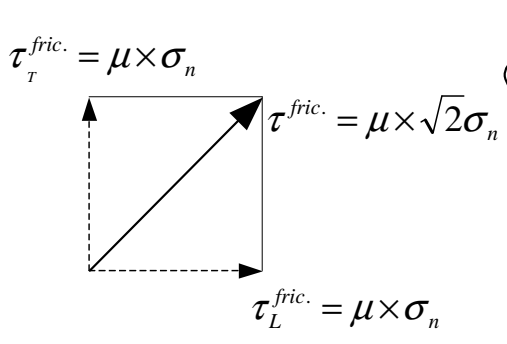

(a)

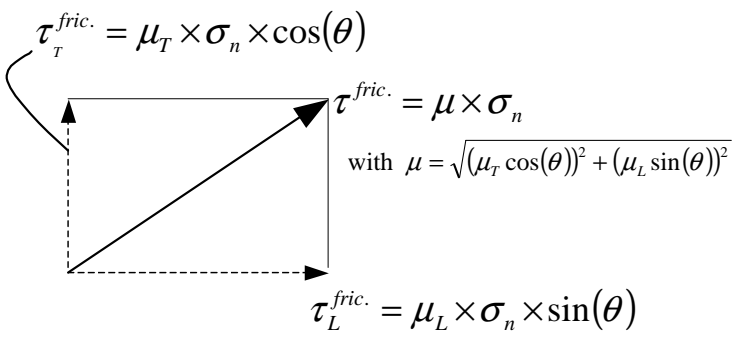

(b)

Fig. 3. (a) Overestimation of the friction stress; (b) model that does not overestimate the friction stresses

LaRC02/03 criterion (Eq. 7). A reasonable model for orthotropic friction is

$$
\left\{\begin{array}{c}
\tau_{T}^{\text {fric. }} \\
\tau_{L}^{\text {fric. }}
\end{array}\right\}=\sigma_{n}\left[\begin{array}{cc}
\mu_{T} & 0 \\
0 & \mu_{L}
\end{array}\right]\left\{\begin{array}{c}
\frac{\tau_{T}}{\|\tau\|} \\
\frac{\tau_{L}}{\|\tau\|}
\end{array}\right\} \therefore\left\{\begin{array}{l}
\tau_{T}^{\text {fric. }}=\sigma_{n} \mu_{T} \cos (\theta) \\
\tau_{L}^{\text {fric. }}=\sigma_{n} \mu_{L} \sin (\theta)
\end{array}\right.
$$

where $\theta$ is the angle formed by the shear-traction vector $\tau$ and the transverse direction in the fracture plane, i.e., $\theta=\arctan \left(\tau_{L} / \tau_{T}\right)$. Curiously, modifying 
LaRC02/03 criterion (Eq. 7) to account for this more representative friction stress, i.e., using

$$
f_{m c}=\left(\frac{\left\langle\left|\tau_{T}\right|+\mu_{T} \sigma_{n} \cos (\theta)\right\rangle}{S_{T}}\right)^{2}+\left(\frac{\left\langle\left|\tau_{L}\right|+\mu_{L} \sigma_{n} \sin (\theta)\right\rangle}{S_{L}}\right)^{2}=1
$$

as a failure criterion yields an envelope that is coincident with Puck's initial criterion (Eq. 6), see Fig. 2.

Also shown in Fig. 2 is the much simpler Sun et al. [15] criterion,

$$
f_{m c}=\left(\frac{\sigma_{b}}{Y_{c}}\right)^{2}+\left(\frac{\tau_{a b}}{S_{L}-\mu_{L} \sigma_{b}}\right)^{2}=1
$$

which exhibits the correct trend.

The use of one of Eqs. 6, 7, 8, and 11 for the failure criterion implies the use of the set of Eqs. 2 for the transformation of stresses. In turn, this means that the fracture angle has to be known. Puck and Schürmann [7] have performed an analytical deduction for Eq. 8 in a plane stress state, but it is much more complex to do so for Eqs. 6, 7 and 11 for 3D stress states. However, Dávila et al. [9] have shown that it is possible to draw the envelope with reasonable accuracy by using a very small number of trial angles. The latter approach is followed in this work.

\subsection{Selection of a matrix compression failure criterion}

The criterion expressed by Eq. 8 is a modified version of the one expressed by Eq. 6 and the failure envelopes for the criteria are almost identical, as shown in Fig. 2. An advantage of the former over the latter consists on the possibility of calculating the fracture angle $\phi$ for each load situation - in plane stress.

For a 3D situation, the advantage does not exist, but the modification intro- 
duced means Eq. 8 does not correctly represent the Mohr-Coulomb criterion. Therefore, for 3D applications, Eq. 6 is preferred over Eq. 8.

As discussed, Eq. 7 over-estimates the friction stresses. Correcting this results in Eq. 11, which correctly applies the Mohr-Coulomb criterion, assuming that the compression reduces the shear stresses. Assuming instead that compression increases the strength results in Eq. 6. Both Eqs. 6 and 11 are physically sound.

Finally, since Eqs. 6 and 11 yield similar results, but the former is simpler, Eq. 6 is selected as the matrix compression failure criterion (i.e. for $\sigma_{n}<0$ ) in this paper.

\section{Matrix tensile failure}

It can be concluded from the WWFE's experimental results [4], that a quadratic interaction between the transverse stress $\sigma_{b}$ and the in-plane shear stress $\tau_{a b}$ describes appropriately the $\left(\sigma_{b}, \tau_{a b}\right)$ failure envelope, for the matrix tensile failure mode. Dávila et al. [9] used this interaction criterion for the LaRC02 failure criteria. However, for the $\left(\sigma_{b}, \tau_{a b}\right)$ failure envelope, the fracture plane is always parallel to the $(a, c)$ plane.

For a generic stress state, the matrix tensile fracture plane does not coincide necessarily with the $(a, c)$ plane. Therefore, it seems reasonable to recast the tractions expressed in the potential fracture planes - as expressed in Eq. 2and apply a quadratic interaction as follows:

$$
f_{m t}=\left(\frac{\sigma_{n}}{Y_{t}}\right)^{2}+\left(\frac{\tau_{T}}{S_{T}}\right)^{2}+\left(\frac{\tau_{L}}{S_{L}}\right)^{2}=1
$$

The criterion applies for $\sigma_{n} \geq 0$, and $Y_{t}$ is the in-plane transverse tensile 


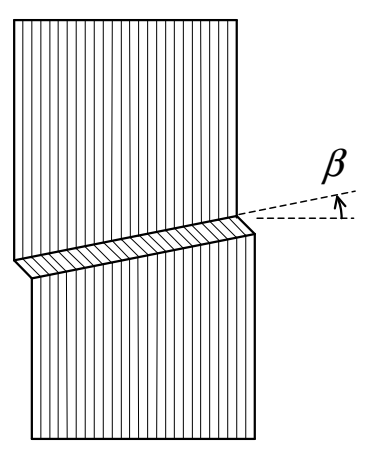

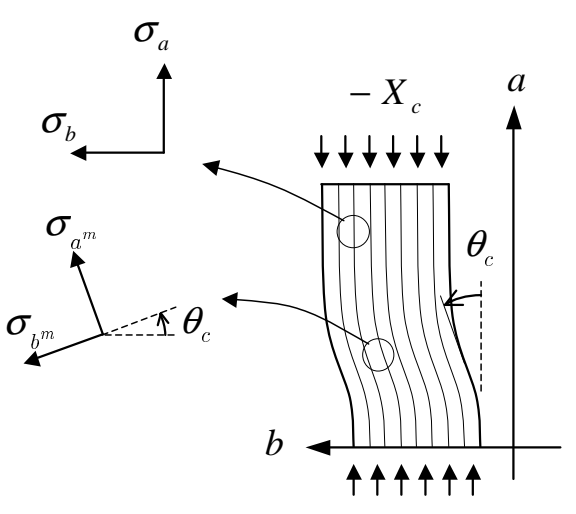

(a)

(b)

$-X_{c}$

Fig. 4. (a) Kinking band; (b) fibre misalignment frame

strength.

\section{Fibre-kinking failure}

In their state of the art review, Schultheisz and Waas [16] conclude that the experimental observations on the kinking phenomenon (Fig. 4(a)) tend to support the contention that kink bands seen in axial compression of high fibre-volume-fraction advanced composite materials occur via a mechanism that is different from the global microbuckling mode suggested by Rosen [17]. Furthermore, they conclude that in advanced composites, kinking seems to be initiated by local microstructural defects, such as fibre misalignments and longitudinal (matrix or interfacial) cracking; therefore, the phenomenon is better understood by treating the problem of stress redistribution, including dynamics, and including both nonlinear geometry and material response.

For the above reasons, a 3D kinking model is now proposed, as a generalization of a 2D kinking model. These are based on Argon's [18] approach and the latter developments by Dávila et al. $[9,10]$. 


\subsection{D kinking model}

Argon [18] assumed that an initial fibre misalignment exists in the composite, which leads to shearing stresses between the fibres. The shearing stresses would act as to further rotate the fibres, which would in turn lead to further increase in the shear stresses. This 'closed loop' effect could then lead to failure. The main result from his analysis is the relation between the compression failure stress, $X_{c}$, the matrix longitudinal shear failure stress, $S_{L}$, and the initial fibre misalignment angle $\theta_{i}$ (in radians):

$$
X_{c}=\frac{S_{L}}{\theta_{i}} .
$$

Budiansky [19] later extended Argon's analysis to

$$
X_{c}=\frac{S_{L}}{\theta_{i}+\gamma^{o}}
$$

where $\gamma^{o}$ is the shear strain at failure. Failure occurs when the shear failure stress is reached in the material coordinate system. In a later analytical work, Budiansky and Fleck [20] included the effect of strain-hardening, shear loads, kink-band inclination and finite fibre stiffness, obtaining a non closedform solution. Another similar solution was obtained assuming kinking in the transverse direction [21] (but not for any generic direction). Analytical models for kink-band broadening were also developed [21]. Kyriakides et al. [22] carried micro-mechanical 2D FE models of the kinking process (modelling fibre and matrix individually), including matrix nonlinearity and initial imperfections. The micro-mechanical 2D FE models were successfully used to predict the propagation stress during kink band broadening [23,24].

More recently, Dávila et al. $[9,10]$ used a very interesting combination of Ar- 
gon's approach [18] and the LaRC02/03 matrix failure criterion. Essentially, Dávila et al. suppose that the fibres might be misaligned, and that further rotation will occur during compressive loading. They then compute the stresses in the updated misalignment frame and check for matrix failure using LaRC02/03 matrix failure criterion.

In the following, the subscript $m$ applied to the shear stress and shear strain designates the misalignment frame at failure, and the subscript $m c$ (also applied to the shear stress and shear strain) designates the misalignment frame at failure for pure axial compression.

Consider a unidirectional composite with a misaligned region being compressed, as depicted in Fig. 4(b). The stresses in the misalignment frame are, for a generic plane stress loading

$$
\begin{aligned}
& \sigma_{a^{m}}=\frac{\sigma_{a}+\sigma_{b}}{2}+\frac{\sigma_{a}-\sigma_{b}}{2} \cos (2 \theta)+\tau_{a b} \sin (2 \theta) \\
& \sigma_{b^{m}}=\frac{\sigma_{a}+\sigma_{b}}{2}-\frac{\sigma_{a}-\sigma_{b}}{2} \cos (2 \theta)-\tau_{a b} \sin (2 \theta) \\
& \tau_{a^{m} b^{m}}=-\frac{\sigma_{a}-\sigma_{b}}{2} \sin (2 \theta)+\tau_{a b} \cos (2 \theta) .
\end{aligned}
$$

For failure under pure compression $\left(\sigma_{a}=-X_{c}, \sigma_{b} \equiv \tau_{a b}=0\right)$, Eqs. 16 lead to $\sigma_{a^{m}}=-X_{c} \cos ^{2}(\theta), \sigma_{b^{m}}=-X_{c} \sin ^{2}(\theta)$ and $\tau_{a^{m} b^{m}}=\tau_{m c}=X_{c} \sin (\theta) \cos (\theta)$.

This stress state can now be placed in an appropriate matrix failure criterion. Using Puck's initial criterion [7] or LaRC02/3 [9,10], Eqs. 6 and 7 respectively (they yield the same result in this case), gives the expression for the specific value of the misalignment angle $\theta$ at failure for a pure compression case $-\theta_{c}$ :

$$
X_{c}\left(\sin \left(\theta_{c}\right) \cos \left(\theta_{c}\right)-\mu_{L} \sin ^{2}\left(\theta_{c}\right)\right)=S_{L}
$$


This angle, $\theta_{c}$, is the sum of the initial misalignment and the rotation due to loading. Dávila et al. [9] have solved Eq. 18 for $\theta_{c}$ resulting in

$$
\theta_{c}=\arctan \left(\frac{1-\sqrt{1-4\left(\frac{S_{L}}{X_{c}}+\mu_{L}\right) \frac{S_{L}}{X_{c}}}}{2\left(\frac{S_{L}}{X_{c}}+\mu_{L}\right)}\right) .
$$

Dávila et al. pointed out that neglecting $\mu_{L}$ and the shear strain, and assuming $\theta_{c}$ to be small (in Eq. 18) yields Argon's equation (Eq. 14). In fact, assuming all the above but now not neglecting the shear strain yields Budiansky's Eq. 15.

Using the shear constitutive law, the shear strain $\gamma_{m c}$ can be obtained from the shear stress $\tau_{m c}$ and so the initial misalignment angle, $\theta_{i}$, can be calculated. In practice, the shear constitutive law is usually nonlinear so that the shear strain can be related to the shear stress by the generic function $f_{C L}$ such that $\tau=f_{C L}(\gamma)$. From the constitutive law, the shear stress at failure (and in the material axes) is a function of the shear strain

$$
\tau_{m c}=f_{C L}\left(\gamma_{m c}\right)
$$

and from the transformation equations (Eqs. 17), the shear stress at an angle $\theta_{c}$ is

$$
\tau_{m c}=\frac{1}{2} \sin \left(2 \theta_{c}\right) X_{c}
$$

From Eqs. 20 and 21, the shear strain at failure for a pure axial compression case, $\gamma_{m c}$, comes as

$$
\gamma_{m c}=f_{C L}^{-1}\left(\frac{1}{2} \sin \left(2 \theta_{c}\right) X_{c}\right)
$$

For instance, for a material which is linear in shear, Eq. 22 becomes simply

$$
\gamma_{m c}=\frac{\sin \left(2 \theta_{c}\right) X_{c}}{2 G_{a b}}
$$

Dávila et al. [9] assumed small angle approximations and reached a simpler 
expression for Eq. 23:

$$
\gamma_{m c}=\frac{\theta_{c} X_{c}}{G_{a b}}
$$

The initial misalignment angle can then be calculated using

$$
\theta_{i}=\theta_{c}-\gamma_{m c}
$$

where $\gamma_{m c}$ can be defined by Eqs. 22, 23 or 24 .

The initial misalignment angle $\theta_{i}$ is a material property, and could be regarded as an equivalent angle that embodies microstructural defects (that can trigger kink-band formation) as well as the actual initial misalignment, like oscillations in the fibre volume fraction or in the bonding to the resin, or microcracks in the resin. Knowing $\theta_{i}$ allows the establishment of an equation defining the shear strain in the material axes for a generic plane stress situation, $\gamma_{m}$, by using the transformation Eqs. 16 and the shear law:

$$
f_{C L}\left(\gamma_{m}\right)=-\frac{\sigma_{a}-\sigma_{b}}{2} \sin \left(2\left(\theta_{i}+\gamma_{m}\right)\right)+\left|\tau_{a b}\right| \cos \left(2\left(\theta_{i}+\gamma_{m}\right)\right)
$$

Having solved the previous equation for $\gamma_{m}$, the misalignment angle $\theta$ come then as

$$
\theta=\frac{\tau_{a b}}{\left|\tau_{a b}\right|}\left(\theta_{i}+\gamma_{m}\right)
$$

Note that, in Eq. 26, a modulus was applied to $\tau_{a b}$ because it is the easiest way of considering simultaneously the possibility of an initial misalignment $\pm \theta_{i}$

For a nonlinear shear response, Eq. 26 can be solved by an iterative process to yield $\gamma_{m}$. For most practical cases, Eq. 26 can be simplified without significant error by assuming small angle approximations:

$$
f_{C L}\left(\gamma_{m}\right) \approx\left(\theta_{i}+\gamma_{m}\right)\left(-\sigma_{a}+\sigma_{b}\right)+\left|\tau_{a b}\right|
$$


For a linear shear behaviour, Eq. 28 can be solved [9], resulting in

$$
\gamma_{m}=\frac{\theta_{i} G_{a b}+\left|\tau_{a b}\right|}{G_{a b}+\sigma_{a}-\sigma_{b}}-\theta_{i}
$$

However, for a nonlinear shear behaviour, there might be no easy way of solving Eq. 28 or 26 without iterating. Having determined the misalignment frame, the stresses can be rotated to that frame, and a matrix failure criterion can be used to check for possible kink-band formation. Dávila et al. $[9,10]$ applied the LaRC02/03 matrix failure criterion to predict failure.

A feature of this model, when a nonlinear shear response is considered, has to do with the possibility of failure by instability, rather than by matrix cracking. To visualize this, lets plot the left hand side and the right hand side of Eq. 26 as a function of $\gamma_{m}$, for the material considered in Table 1 in a pure axial compression situation, Fig. 5, considering linear and nonlinear shear responses. In Fig. 5(a), a compressive stress equal to the compressive strength is applied (800 MPa) and there is an equilibrium point for both the linear and the nonlinear shear behaviour. In Fig. 5(b), the compressive stress is increased to $970 \mathrm{MPa}$, and there is no equilibrium solution for the nonlinear shear behaviour. Note that this is not due to the fact that the compressive strength has been exceeded, since the same could have been achieved by maintaining the applied stress and changing the nonlinear shear response.

The physical interpretation for this phenomenon is that, for a nonlinear shear behaviour, failure might occur not only by matrix cracking, but also as the result of an elastic instability. The instability arises when the curves corresponding to the left and right hand side of Eq. 26 are tangential to each other. This is because, if the two curves are tangential to each other, any increase in the compressive stress will lead to a situation of non-interception, 


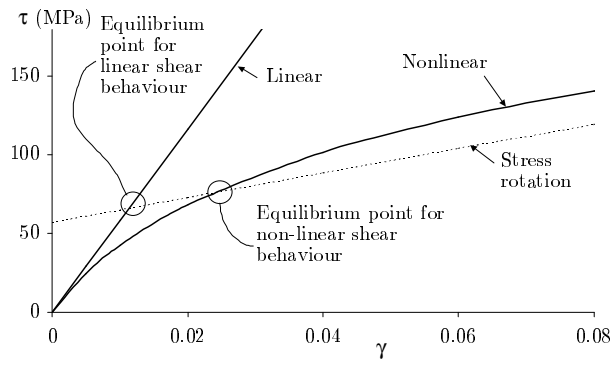

(a)

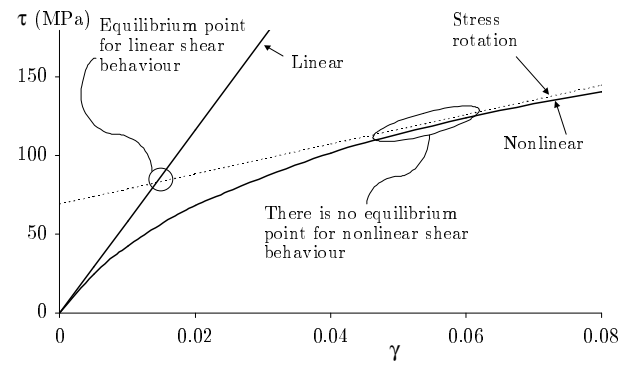

(b)

Fig. 5. (a) Equilibrium point for a linear and a nonlinear material in shear; (b) equilibrium only for the linear shear behaviour and thus in unlimited rotation of the fibres (instability). The stress states and corresponding shear strain corresponding to failure by instability can thus be mathematically defined by the system

$$
\left\{\begin{array}{c}
f_{C L}\left(\gamma_{m}\right)=-\frac{\sigma_{a}-\sigma_{b}}{2} \sin \left(2\left(\theta_{i}+\gamma_{m}\right)\right)+\left|\tau_{a b}\right| \cos \left(2\left(\theta_{i}+\gamma_{m}\right)\right) \\
\frac{\partial f_{C L}\left(\gamma_{m}\right)}{\partial \gamma_{m}}=-\left(\sigma_{a}-\sigma_{b}\right) \cos \left(2\left(\theta_{i}+\gamma_{m}\right)\right)-2\left|\tau_{a b}\right| \sin \left(2\left(\theta_{i}+\gamma_{m}\right)\right)
\end{array}\right.
$$

This system (Eq. 30) defines an envelope for failure by instability. Fibre kinking is thus predicted not only if the matrix failure criterion (in the misalignment frame) is verified, but also if the system (Eq. 30) is verified.

\subsection{Proposed 3D kinking model}

Most fibre-kinking models assume that kinking happens in the plane of the lamina. On the other hand, most experimental studies mimic this in-plane approach and constrain the specimens so that out of plane movements are not allowed. However, many researchers agree on the 3D nature of fibre-kinking failure. A 3D kinking model based on the previous 2D model is now proposed. 
This model assumes initial fibre misalignment and nonlinear shear behaviour. Furthermore, its formulation is such that an efficient numerical FE implementation is possible.

Consider a unidirectional lamina under a general compressive stress state as shown in Fig. 6(a). The fibre-kinking plane is assumed to be at an angle $\psi$ with the $b$ axis, as shown in Fig. 6(b). Fig. 6(c) shows the stresses acting on the $(b, c)$ plane, while Fig. $6(\mathrm{~d})$ shows the stresses acting along the $b^{\psi}$ and $c^{\psi}$ directions (Fig. 6(d) assumes that $b^{\psi}$ and $c^{\psi}$ are the principal directions in the plane $(b, c))$. The rotation to the misalignment plane is shown in Fig. 6(e). The matrix fracture plane is represented in Fig. 6(f). The value of the angle $\psi$ depends on the particular stress state ${ }^{2}$. A 2D fibre-kinking model-in which through-the-thickness movements are constrained-assumes that the angle $\psi$ in Figs. 6(b), (c) and (d) is equal to zero. If the composite is constrained so that it cannot move in the $b$ direction, then the fibre-kinking plane would have an angle $\psi=90^{\circ}$. For a general load situation, $\psi$ will have a value between 0 and $180^{\circ}$.

The set of transformation Eqs. 31 can now be used to rotate the stresses to

$\overline{2}$ In reality, defects such as fibre initial misalignment may not be homogeneously distributed and the kinking plane could in fact also be influenced by that 
the fibre-kinking plane:

$$
\begin{aligned}
& \sigma_{b^{\psi}}=\frac{\sigma_{b}+\sigma_{c}}{2}+\frac{\sigma_{b}-\sigma_{c}}{2} \cos (2 \psi)+\tau_{b c} \sin (2 \psi) \\
& \sigma_{c^{\psi}}=\sigma_{b}+\sigma_{c}-\sigma_{b^{\psi}} \\
& \tau_{a b^{\psi}}=\tau_{a b} \cos (\psi)+\tau_{c a} \sin (\psi) \\
& \tau_{b^{\psi} c^{\psi}}=-\frac{\sigma_{b}-\sigma_{c}}{2} \sin (2 \psi)+\tau_{b c} \cos (2 \psi) \\
& \tau_{c^{\psi} a}=\tau_{c a} \cos (\psi)-\tau_{a b} \sin (\psi) .
\end{aligned}
$$

After defining the fibre-kinking plane, the stresses are then rotated to the misalignment frame. The strain $\gamma_{m}$ is obtained by solving the iterative equation

$$
f_{C L}\left(\gamma_{m}\right)=-\frac{\sigma_{a}-\sigma_{b^{\psi}}}{2} \sin \left(2\left(\theta_{i}+\gamma_{m}\right)\right)+\left|\tau_{a b^{\psi}}\right| \cos \left(2\left(\theta_{i}+\gamma_{m}\right)\right)
$$

and the angle $\theta$ come as

$$
\theta=\frac{\tau_{a b^{\psi}}}{\left|\tau_{a b^{\psi}}\right|}\left(\theta_{i}+\gamma_{m}\right)
$$

Having established the orientation of the misalignment frame, the stresses can be rotated to it using

$$
\begin{aligned}
& \sigma_{a^{m}}=\frac{\sigma_{a}+\sigma_{b^{\psi}}}{2}+\frac{\sigma_{a}-\sigma_{b^{\psi}}}{2} \cos (2 \theta)+\tau_{a b^{\psi}} \sin (2 \theta) \\
& \sigma_{b^{m}}=\sigma_{a}+\sigma_{b^{\psi}}-\sigma_{a^{m}} \\
& \tau_{a^{m} b^{m}}=-\frac{\sigma_{a}-\sigma_{b^{\psi}}}{2} \sin (2 \theta)+\tau_{a b^{\psi}} \cos (2 \theta) \\
& \tau_{b^{m} c^{\psi}}=\tau_{b^{\psi} c^{\psi}} \cos (\theta)-\tau_{c^{\psi} a} \sin (\theta) \\
& \tau_{c^{\psi} a^{m}}=\tau_{c^{\psi} a^{\psi}} \cos (\theta) .
\end{aligned}
$$

At this point, a check can be performed for matrix failure. For compression 
$\left(\sigma_{b^{m}} \leq 0\right)$, one can apply the already presented matrix compression failure criterion (Eqs. 6 and 30), while for tensile $\left(\sigma_{b^{m}}>0\right)$ the matrix tensile failure criterion (Eq. 13) is applied. The criterion for kinking comes then as:

for $\sigma_{b^{m}} \leq 0$

$$
\begin{gathered}
f_{k i n k}=\left(\frac{\tau_{T}}{S_{T}-\mu_{T} \sigma_{n}}\right)^{2}+\left(\frac{\tau_{L}}{S_{L}-\mu_{L} \sigma_{n}}\right)^{2}=1 \\
\text { or } \\
\left\{\begin{array}{c}
f_{C L}\left(\gamma_{m}\right)=-\frac{\sigma_{a}-\sigma_{b^{\psi}}}{2} \sin \left(2\left(\theta_{i}+\gamma_{m}\right)\right)+\left|\tau_{a b^{\psi}}\right| \cos \left(2\left(\theta_{i}+\gamma_{m}\right)\right) \\
\frac{\partial f_{C L}\left(\gamma_{m}\right)}{\partial \gamma_{m}}=-\left(\sigma_{a}-\sigma_{b^{\psi}}\right) \cos \left(2\left(\theta_{i}+\gamma_{m}\right)\right)-2\left|\tau_{a b^{\psi}}\right| \sin \left(2\left(\theta_{i}+\gamma_{m}\right)\right)
\end{array}\right.
\end{gathered}
$$

for $\sigma_{b^{m}}>0$

$$
f_{k i n k}=\left(\frac{\sigma_{n}}{Y_{t}}\right)^{2}+\left(\frac{\tau_{T}}{S_{T}}\right)^{2}+\left(\frac{\tau_{L}}{S_{L}}\right)^{2}=1
$$

In Eq. 35, the tractions in the fracture plane are given by

$$
\begin{aligned}
& \sigma_{n}=\frac{\sigma_{b^{m}}+\sigma_{c^{\psi}}}{2}+\frac{\sigma_{b^{m}}-\sigma_{c^{\psi}}}{2} \cos (2 \phi)+\tau_{b^{m} c^{\psi}} \sin (2 \phi) \\
& \tau_{T}=-\frac{\sigma_{b^{m}}-\sigma_{c^{\psi}}}{2} \sin (2 \phi)+\tau_{b^{m} c^{\psi}} \cos (2 \phi) \\
& \tau_{L}=\tau_{a^{m} b^{m}} \cos (\phi)+\tau_{c^{\psi} a^{m}} \sin (\phi)
\end{aligned}
$$

where the angle $\phi$ is obtained by trying a small number of tentative angles in the interval $0 \leq \phi<\pi$, and the angle $\psi$ has yet to be determined. A possible solution to determine $\psi$ is to apply the criterion expressed in Eqs. 35, 36 and 37 to a range of tentative angles $\psi$ in the interval $0 \leq \psi<\pi$. For a numerical implementation of the criterion however, the numerical effort dispensed could be cumbersome. It is now shown that it is possible to define a pragmatic expression for $\psi$. 
The analysis of specimens failed by kink-band formation shows that, in the kink band, the fibres rotate consistently in the same direction forming planes of kinked fibres. Consider now an element of volume of a composite, under a generic loading as in Fig. 6(a). The stresses acting on a section in the $(b, c)$ plane are shown in Fig. 6(c). Suppose that the element of volume fails by the formation of a kink band in its interior; a plane of kinked fibres is shown in Fig. 6(b). During (and after) the failure process, the shear stiffness in the failed band is lower than outside it. As a result, if the shear stress $\tau_{b^{\psi}} c^{\psi}$ is nonzero, the lower shear stiffness in the kink band would result in an out-ofplane movement of the kinked fibres, which contradicts the assumption that $\left(b^{\psi}, c^{\psi}\right)$ is the plane where fibre kinking takes place. Therefore, $\tau_{b^{\psi}} c^{\psi}$ has to be zero, and $b^{\psi}$ and $c^{\psi}$ are thus the principal directions in the plane $(b, c)$, Fig. $6(d)$.

Another argument leading to the same conclusion is that a negative stress $\sigma_{b} \psi$ will tend to close any micro-cracks in the matrix (normal to the $b^{\psi}$ direction) disfavouring fibre kinking in that direction, while a positive stress $\sigma_{b^{\psi}}$ will tend to open those same micro-cracks, now favouring fibre kinking. Hence, it is reasonable to expect that the $b^{\psi}$ is the direction corresponding to maximum principal stress in the plane $(b, c)$. Finally, the angle $\psi$ is thus given by

$$
\tan (2 \psi)=\frac{2 \tau_{b c}}{\sigma_{b}-\sigma_{c}}
$$

To conclude, note that the laminated construction may lead to different fibre arrangements within the plane of a lamina and in the through-the-thickness direction. Initial fibre misalignment angles have been reported to be smaller in the through-the-thickness direction [25], but more recent results suggest they 


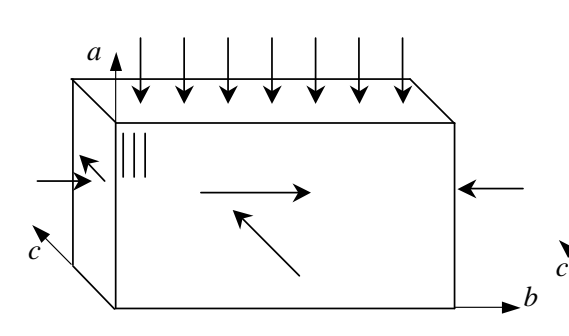

(a)

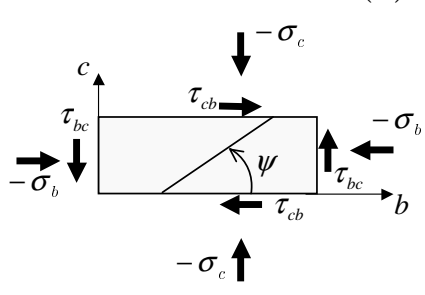

(c)

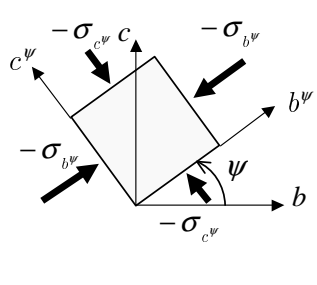

(d)

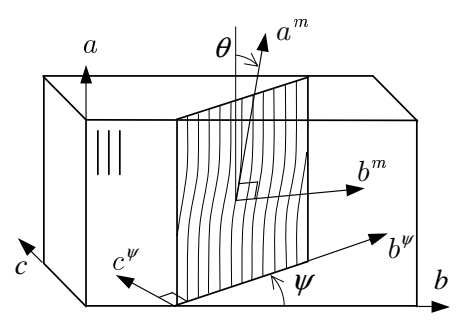

(b)

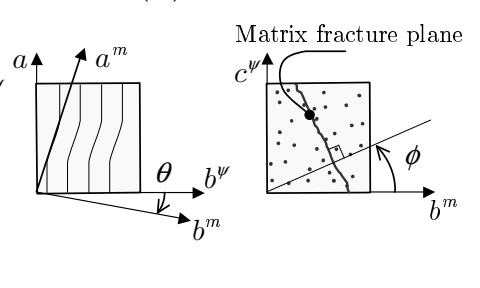

(f)

Fig. 6. 3D kinking model

can be similar in magnitude [26]. The present model assumes that the initial fibre misalignment is equal in magnitude in the transverse direction, through the thickness direction, or in any direction between the two. In order to apply this model, the only material properties that need to be known, in addition to the in-plane shear response, are $Y_{c}, S_{L}, \phi_{o}, Y_{t}$ and $X_{c}$. All the remaining parameters follow from these.

\section{Applications}

\subsection{Failure envelope $\left(\sigma_{b}, \tau_{a b}\right)$}

The matrix failure model (compression and tension) is here used to predict the $\left(\sigma_{b}, \tau_{a b}\right)$ failure envelope for an unidirectional composite E-glass/LY556. Material properties, Table 2, and experimental data, Fig. 7, are given by Soden et al. [4,27]. Since more than one value is reported for the transverse (compressive and tensile) and shear strengths, the corresponding average values are 
Table 2

Mechanical properties of a unidirectional E-glass/LY556

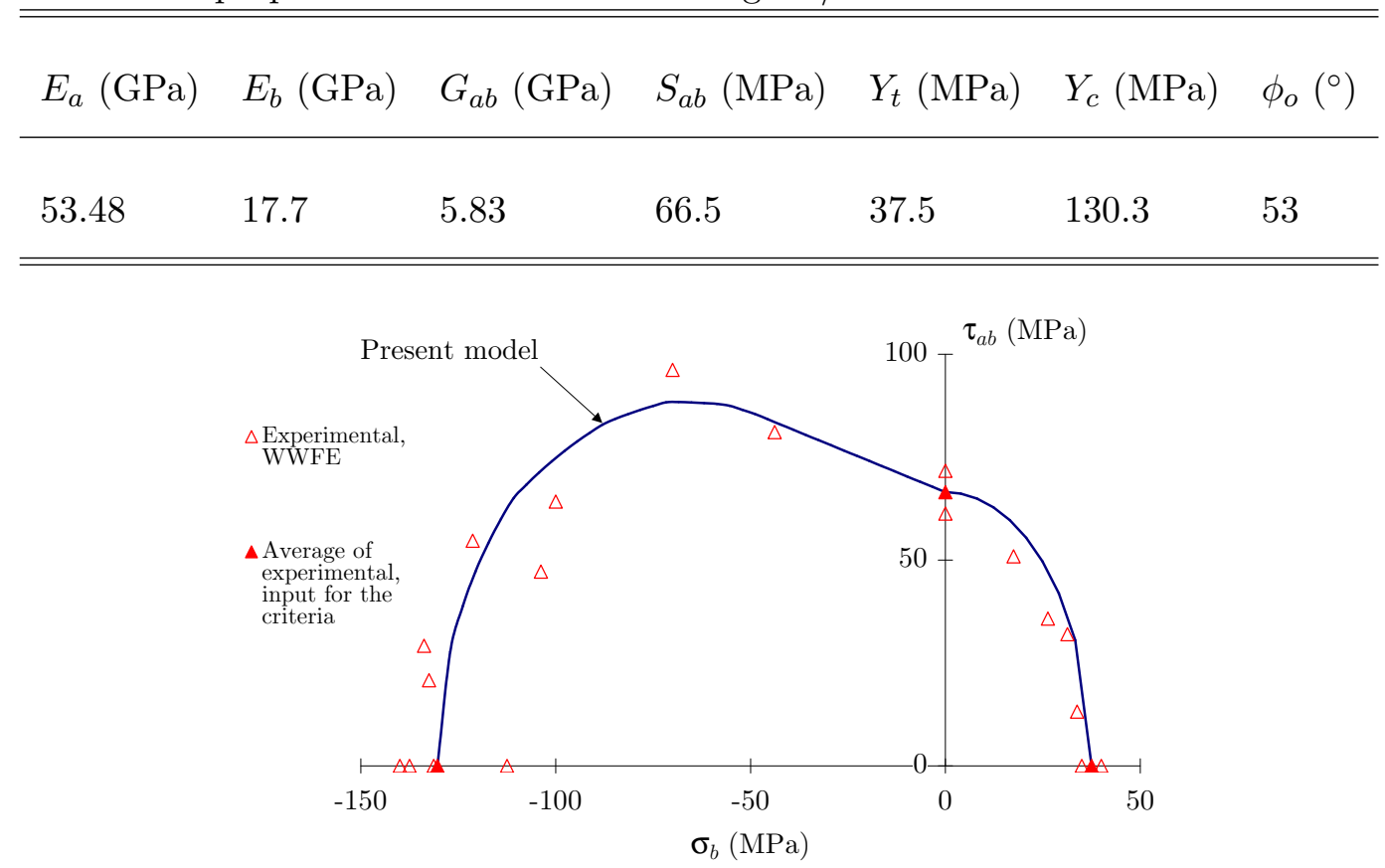

Fig. 7. Failure envelopes and WWFE test data for unidirectional composite E-Glass/LY556

used in all models.

The envelope predicted by the model is given in Fig. 7, where a good correlation with the experimental results can be observed.

\subsection{Axial compression with superposed hydrostatic pressure}

Unfortunately, there is not much experimental data on fibre kinking under a multi-axial stress state. One exception is the compressive behaviour of composite rods, with superposed hydrostatic pressure. Wronsky and Parry [28] measured the effect of hydrostatic pressure on the compressive strength for a GRP composite. The compressive strength without superposed hydrostatic pressure was measured as $1150 \mathrm{MPa}$. Three values of shear strength are re- 


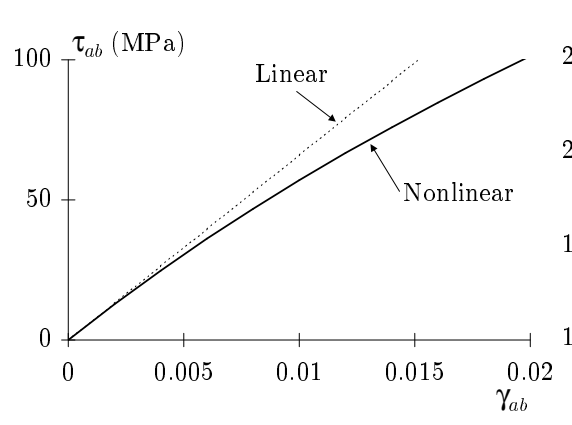

(a)

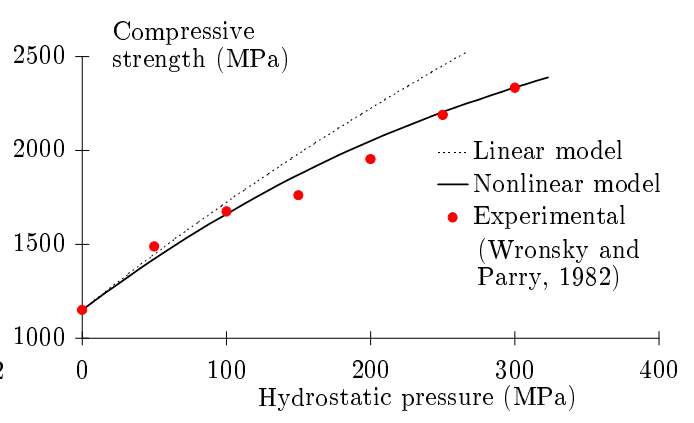

(b)

Fig. 8. (a) linear and nonlinear shear curves considered; (b) compressive strength as a function of the hydrostatic pressure

ported, depending on the test method and specimen dimensions, 42, 48 and $59 \mathrm{MPa}$. The biggest value of the three is used herein, for being (arguably) more representative.

These material properties are not enough to completely define the material for the purposes of the failure model. Therefore, some material properties have been assumed from typical values as follows: (i) the shear modulus is taken as $6600 \mathrm{MPa}$, (ii) the fracture angle in matrix compression is $\phi_{o}=53^{\circ}$, (iii) the transverse compressive strength is taken as $140 \mathrm{MPa}$. To analyse the effect of shear nonlinearity, a logarithmic law is considered, $\tau=k_{1} \ln \left(k_{2} \gamma+1\right)$, with $k_{1}=200 \mathrm{MPa}$ and $k_{2}=33$, which yields the same initial shear modulus. The linear and nonlinear curves are compared in Fig. 8(a).

The effect of the hydrostatic pressure on the compressive strength, as predicted from the model, is compared with the experimental data from Wronsky and Parry [28] in Fig. 8(b). The comparison suggests that the physics of the compressive behaviour may have been correctly represented in the model, but clearly, experimental measurements of the assumed material properties are required for a rigorous validation of the model. 


\subsection{Cross-ply laminate}

A last application example is presented, which consists of the compression of carbon-epoxy AS4/3502 laminates with lay-up $( \pm \theta)_{n s}$. For these laminates, the failure can be matrix-dominated or fibre-dominated depending on the angle

$\theta$. The material properties used by the model are from Shuart [26]: $E_{a}=$ $127.6 \mathrm{GPa}, E_{b}=11.3 \mathrm{GPa}, G_{a b}=6.0 \mathrm{GPa}, \nu_{a b}=0.3, X_{c}=1045 \mathrm{MPa}, S_{L}=$ $95 \mathrm{MPa}$ and the shear strain at failure is $4 \%$. In addition to these properties, the model also requires the fracture angle in pure transverse compression, which is considered to be $\phi_{o}=53^{\circ}$. For the non-linear shear behaviour, the logarithmic law is considered, $\tau=k_{1} \ln \left(k_{2} \gamma+1\right)$, with the constants $k_{1}$ and $k_{2}$ computed such that the non-linear curve has the same initial slope as the linear one, and that it passes through the point $(\gamma=0.04, \tau=95)$, which yields $k_{1}=58.2 \mathrm{MPa}$ and $k_{2}=103.1$. The linear and non-linear shear curves are shown in Fig. 9(a) and the predictions from the model are shown to be in good agreement with the experimental data, Fig. 9(b). For this application, the influence of the non-linear shear behaviour does not appear to be particularly important.

\section{Conclusions}

The criteria proposed, and the physically-based models developed, are shown to accurately predict failure envelopes and trends. The fibre compression failure criterion proposed emphasizes the need for accurate characterization of the shear behaviour. The fibre-kinking model can be readily used in a stochastic formulation, since manufacturing defects can be easily accounted for within 


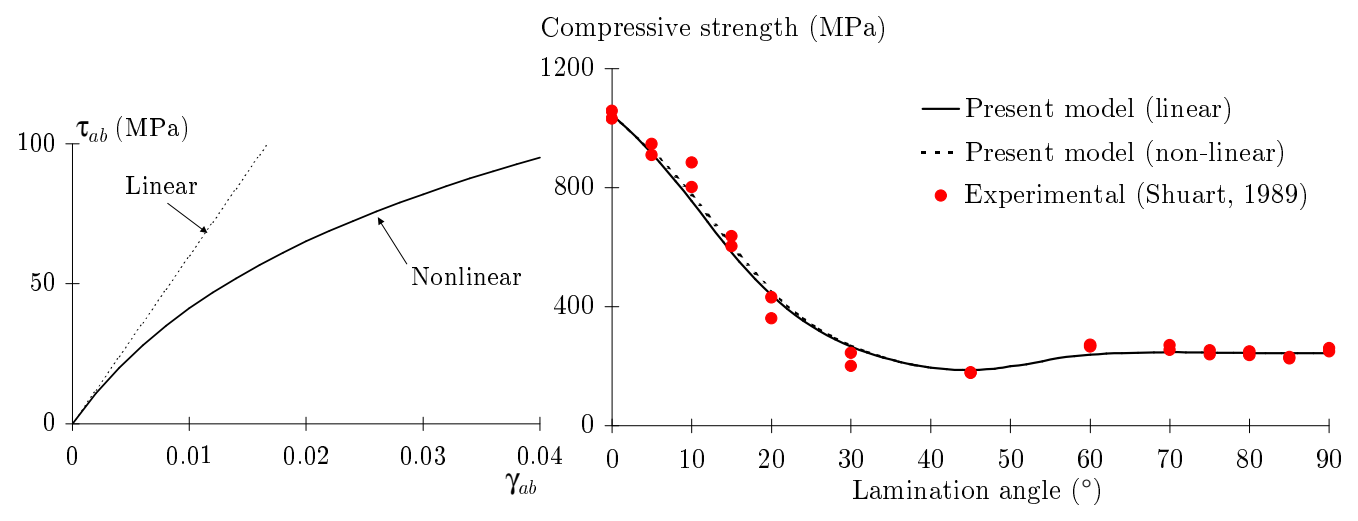

(a)

(b)

Fig. 9. (a) Linear and non-linear shear curves; (b) comparison of the results from the present model to the experimental results from Shuart [26] the model.

The application of these criteria to more complex structures requires the use of numerical methods. An implementation of these criteria in FE is described in the second paper of this series [12].

\section{Acknowledgements}

The funding of this research from the Portuguese Foundation for Science and Technology is greatfully acknowledged.

\section{References}

[1] M. J. Hinton, P. G. Soden, A comparison of the predictive capabilities of current failure theories for composite laminates, judged against experimental evidence, Composites Science and Technology 62 (2002) 1725-1797.

[2] Z. Hashin, A. Rotem, A fatigue failure criterion for fibre reinforced materials, 
Journal of Composite Materials 7 (1973) 448-464.

[3] Z. Hashin, Failure criteria for unidirectional fibre composites, Journal of Applied Mechanics 47 (1980) 329-334.

[4] P. D. Soden, M. J. Hinton, A. S. Kaddour, Biaxial test results for strength and deformation of a range of e-glass and carbon fibre reinforced composite laminates: Failure exercise benchmark data, Composites Science and Technology 62 (2002) 1489-1514.

[5] A. Puck, Calculating the strength of glass fibre/plastic laminates under combined load, Kunststoffe, German Plastics 55 (1969) 18-19, original in German.

[6] A. Puck, W. Schneider, On failure mechanisms and failure criteria of filamentwound glass-fibre/resin composites, Plastics and Polymers (1969) 33-44Original in German.

[7] A. Puck, H. Schürmann, Failure analysis of FRP laminates by means of physically based phenomenological models, Composites Science and Technology 58 (1998) 1045-1067.

[8] A. Puck, H. Schürmann, Failure analysis of FRP laminates by means of physically based phenomenological models, Composites Science and Technology 62 (2002) 1633-1662.

[9] C. G. Dávila, N. Jaunky, S. Goswami, Failure criteria for FRP laminates in plane stress, in: 44th AIAA/ ASME/ ASCE/ AHS/ ASC Structures, Structural Dynamics, and Materials Conference, 2003, AIAA Paper 2003-1991.

[10] C. G. Dávila, P. P. Camanho, Failure criteria for FRP laminates in plane stress, Tech. Rep. NASA/TM-2003-212663, National Aeronautics and Space Administration, U. S. A. (2003). 
[11] S. T. Pinho, L. Iannucci, P. Robinson, Physically-based failure models and criteria for laminated fibre-reinforced composites. Development and Implementation, Tech. Rep. 04-02, Imperial College London, Department of Aeronautics (2004).

[12] S. T. Pinho, L. Iannucci, P. Robinson, Physically-based failure models and criteria for laminated fibre-reinforced composites. Part II: FE implementation, Submitted to Composites: Part A.

[13] Livermore Software Technology Corporation, California, USA, LS-Dyna 970 (2003).

[14] A. Puck, Failure analysis for FRP. Models for use in practice, Hanser, Munich, Viena. Original in German (1996).

[15] C. T. Sun, B. J. Quinn, D. W. Oplinger, Comparative evaluation of failure analysis methods for composite laminates, DOT/FAA/AR (1996) 95-109.

[16] C. R. Schultheisz, A. M. Waas, Compressive failure of composites, part I: Testing and micromechanical theories, Progress in Aerospace Sciences 32 (1996) $1-42$.

[17] V. W. Rosen, Mechanics of composite strengthening, Fiber Composite Materials American Society of Metals, Metals Park, Ohio (1965) 37-75.

[18] A. S. Argon, Fracture of composites, in: Treatise on Materials Science and Technology, Academic Press, New York, 1972, pp. 79-114.

[19] B. Budiansky, Micromechanics, Computers and Structures 16 (1983) 3-12.

[20] B. Budiansky, N. A. Fleck, Compressive failure of fibre composites, Journal of the Mechanics and Physics of Solids 41 (1) (1993) 183-211.

[21] B. Budiansky, N. A. Fleck, J. C. Amazigo, On kink-band propagation in fiber 
composites, Journal of the mechanics and physics of solids 46 (9) (1998) 16371653.

[22] S. Kyriakides, R. Arseculeratne, E. J. Perry, On the compressive failure of fiber reinforced composites, International Journal of Solids and Structures $32(6 / 7)$ (1995) 689-738.

[23] T. J. Vogler, S. Kyriakides, Initiation and axial propagation of kink bands in fiber composites, Acta Materialia 45 (6) (1997) 2443-2454.

[24] T. J. Vogler, S. Kyriakides, On the axial propagation of kink bands in fiber composites: Part i experiments, International Journal of Solids and Structures 36 (1999) 557-574.

[25] J. G. Davis Jr, Compressive strength of fiber-reinforced composite materials, in: Composite Reliability, ASTM STP 580, Americal Society for Testing and Materials, Philadelphia, 1975, pp. 364-377.

[26] M. J. Shuart, Failure of compression-loaded multidirectional composite laminates, AIAA Journal 27 (1989) 1274-1279.

[27] P. D. Soden, M. J. Hinton, A. S. Kaddour, Lamina properties, lay-up configurations and loading conditions for a range of fibre-reinforced composite laminates, Composites Science and Technology 58 (1998) 1011-1022.

[28] A. S. Wronsky, T. V. Parry, Compressive failure and kinking in uniaxially aligned glass-resin composite under superposed hydrostatic pressure, Journal of Materials Science 17 (1982) 3656-3662. 